\title{
Transitional Interface: Concept, Issues and Framework
}

\author{
Raphaël Grasset* Julian Looser ${ }^{\dagger} \quad$ Mark Billinghurst ${ }^{*}$ \\ Hitlab NZ \\ University of Canterbury \\ Private Bag 4800, Christchurch, NZ
}

\begin{abstract}
Transitional Interfaces have emerged as a new way to interact and collaborate between different interaction spaces such as Reality, Virtual Reality and Augmented Reality. In this paper we explore this concept further. We introduce a descriptive model of the concept, its collaborative aspect and how it can be generalized to describe natural and continuous transitions between contexts (e.g. across space, scale, viewpoint, and representation).
\end{abstract}

\section{INTRODUCTION}

Recent work in Augmented Reality (AR) has demonstrated how AR can offer an efficient co-located interacting space, while VR can provide an experience where space and time can be fully controlled.

Allowing users to simultaneously interact in multiple types of spaces (AR,VR) and environments at the same time can be beneficial for a large number of applications such as architecture, chemistry, etc. Although it is possible for collaborators to be present in different spaces, moving between these spaces can also be quite advantageous. In the MagicBook project [1], an interface was proposed to allow seamless transitions between different interaction spaces. A lot of other projects have replicated this idea, but none has tried to formally describe or evaluate the concept. Furthermore, many perceptual and awareness factors have been ignored, such as the user's awareness requirements during a transition.

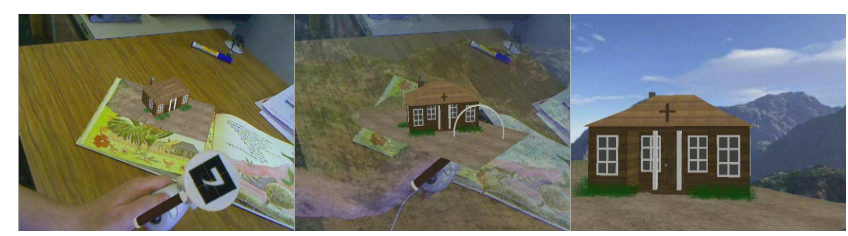

Figure 1: New transitional interface redefined: a continuous perceptual transition between different contexts.

The concept of a transition can be considered in respect to other recent work in Virtual Reality. In fact, multiscale collaboration (such as macro- and micro-scales in chemistry [3]), or accessing multiple viewpoints and representations (such as realistic versus non photo-realistic rendering), can be treated as a similar problem: how do we effectively interact with, transition between, and collaborate across different types of environments?

The lack of formalization or adapted tools motivate us to provide a deeper study of the notion of 'transition'. In this paper we describe an initial model that identifies the main issues of this general problem.

\section{Transitional Concept}

We introduce the notion of Context related to an environment where users can collaborate and interact. A context not only de-

\footnotetext{
*e-mail: raphael.grasset@hitlabnz.org

†e-mail:julian.looser@hitlabnz.org

†-mail:mark billinghurst@hitlabnz.org
}

fines a space (e.g. AR, VR, Reality), but can define a scale (e.g. macro, micro, nano, etc.), a representation (e.g. photorealistic, nonphotorealistic, symbolic), and any other user parameters (such as viewpoints and navigation mode). A context is the collection of values of parameters relevant to the application. For example, one context may be defined as an AR space, on a 1:1 egocentric scale with cartoon rendering and a walking navigation metaphor.

A user can navigate and manipulate content within a context but can also transition to other contexts. Firstly, we present the case of a single-user, followed by a collaborative scenario. For a more general description of the different viewpoint types (exocentric/egocentric, focus/secondary view) and the relationship between the application data and the environment, readers can refer to our previous work [2] where we introduce much of the notation used in this paper.

\subsection{Single-User}

Based on the definition proposed in [2], a transition is a function of the movement between two viewpoints within two distinct contexts 1 . We now extend this definition to cover not only a change in viewpoint, but also the possibility of change in scale, representation and interaction. Therefore, the transition is between entire contexts. A transitional interface is an interaction technique supporting a this concept.

A transition between two contexts can be decomposed into a succession of different actions. Figure 2 describe these steps of navigation and transition between different contexts:

1. User can navigate in the first context based on a locomotion function $V(t)$.

2. User can initiate a transition implicitly (e.g. click on a position on a map, 'selection' mode).

3. User is in a restricted mode where his view "moves" between the two contexts.

4. User is reaching a new context.

5. User can navigate in this new context based on a similar or new locomotion function $V(t)$.

6. User can optionally come back to the first context, by using the same transition function (so we have this notion of 'deselection') or another one. User can therefore come back to his previous state on the other context (e.g. viewpoint) or can also be a new one.

The transition function needs to take user perceptual factors into account. Recent work in this area has been limited to deliberately simple solutions: a brutal disjointed switch between a view of the real world and a black VR background or perhaps a simple linear interpolation between the two viewpoint positions. Previous work on Virtual Reality shown that an application needs to favor a continuously smooth transitional motion (fading) rather than the discontinuous and fast approach of teleportation. We hypothesize that these concepts need to be applied in the case of the transitional interface from a spatial and visual aspect.

\footnotetext{
${ }^{1}$ From an etymological viewpoint, we are using the verb 'to transition' for defining the action, and 'transitioning' for being in this state
} 


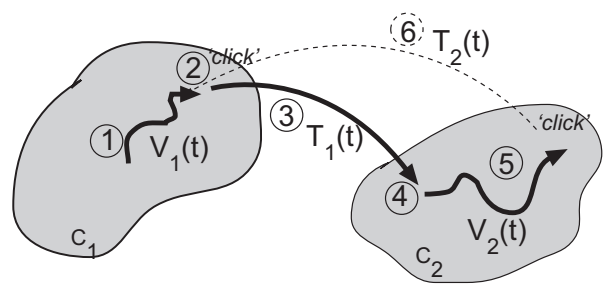

Figure 2: Description of a Transitional Interface

A user needs not only to be able to identify him or herself in the different contexts (such as by seeing a virtual hand in a VR space), but also during the transition. Furthermore, if the representation of the user is very different between contexts, he or she might feel disturbed when transitioning and be disoriented in the new context.

A coherence needs to be maintained between the different representations chosen for the application content within the different contexts ([3]). Respecting logical spatial relationships, pictorial similarity, articulated dimensionality or topology of the object representations are important criterion. Consequently, we can list the different issues that have been identified:

1. Which interaction techniques are used to initiate a transition?

2. Which transition function are used to maintain a seamless spatial and visual representation between the two contexts?

3. How can a sense of proprioception be maintained during the transition?

4. How can the user come back to the previous context? Does the user need to move back to the same location?

5. How can coherence be maintained of the application's content between contexts?

6. How can coherence be maintained of the presence between contexts?

7. How can coherence be maintained in the interaction between contexts?

\subsection{Multi-User}

In the case of a collaborative application, awareness of other people needs to be provided to the users. In the literature, the common parameters cited are generally:

- Who (presence, identity of users),

- What (their intentions, feedthrough of their actions),

- Where (location, gaze, view feedback).

A user is generally embodied as a virtual model replicating their behavior; an avatar. A transitional collaborative interface needs to also provide similar awareness components: between users in a same context (proximal embodiment), between users in different contexts (distal embodiment), and also during a transition step.

Figure 3 illustrates a representative example. In this scenario we have three users: user $A$ and user $B$ are in context $1(C 1)$, while user $C$ is in context $2(C 2)$. We need to maintain awareness cues between users in the same context (user $A$ and user $B$ ), but also a distal embodiment for users in different contexts (user $A$ and user $B$ for user $C$, user $C$ for user $A$ and user $B$ ).

When user $A$ is transitioning between contexts (step 2), other users need to be aware of the transition stage. When the transition is complete, the distal and direct awareness for user $A$ has changed, user $B$ now has a distal embodiment of user $A$ while user $C$ has a proximal embodiment.

We can also list the different new issues identified for the multiuser scenario:
(1)
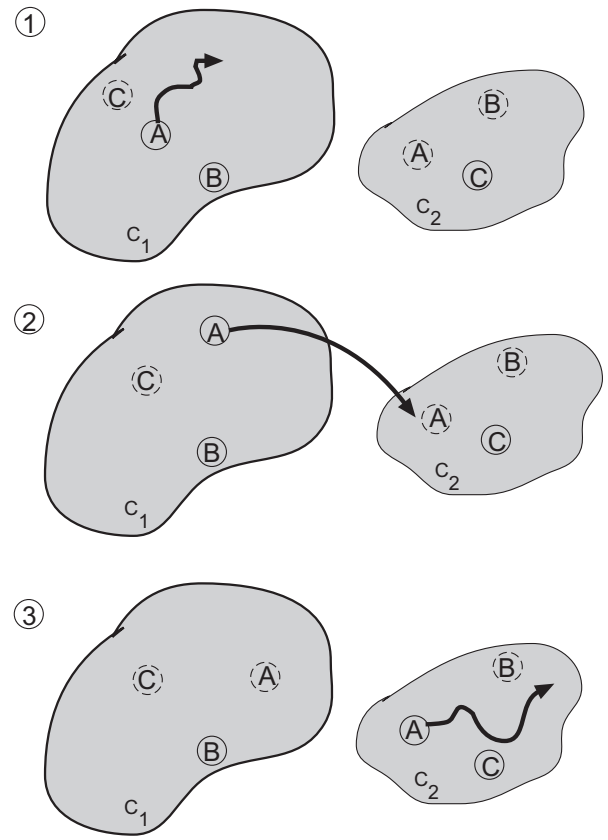

Figure 3: Steps of a user transitioning in a collaborative transitional application (in circle, representation of proximal embodiment, in dot circle, distal embodiment).

1. How to maintain awareness for other users while a user is transitioning between contexts? (from the start, during and the end of the transition)

2. How to illustrate from which context the user is transitioning to and from?

3. How to modify the proximal embodiment to a distal embodiment of a user transitioning?

4. How to maintain co-context and cross-context awareness (co-presence, cross-presence)?

5. How to maintain co-context and cross-context information sharing?

6. How to maintain co-context and cross-context interaction?

\section{Conclusion}

We have presented a full model of the transitional concept and issues related to transitional collaboration. The notion of presence and co-presence needs to be refined and deeply evaluated due to the new issues of transitional collaboration. Also, some research needs to be conducted on how to maintain visual and spatial coherence during a transition between two contexts when we accelerate the movement for better efficiency.

\section{REFERENCES}

[1] Mark Billinghurst, Hirkazu Kato, and Ivan Poupyrev. The magicbook: Moving seamlessly between reality and virtuality. IEEE Computer Graphics and Applications, 21(3):6-8, 2001.

[2] Mark Billinghurst Raphal Grasset, Philip Lamb. Evaluation of mixedspace collaboration. In International Symposium on Mixed and Aug mented Reality (ISMAR05), 2005.

[3] X. Zhang and G. Furnas. mcves: Using cross-scale collaboration to support user interaction with multiscale structures. PRESENCE: Teleoperators and Virtual Environments, 14(1):31-46, February 2005. 